\title{
The price for reduced light toxicity: Do endoilluminator spectral filters decrease color contrast during Brilliant Blue G-assisted chromovitrectomy?
}

\author{
Paul B. Henrich • Christophe Valmaggia • Corina Lang • \\ Philippe C. Cattin
}

Received: 4 May 2013 /Revised: 7 August 2013 / Accepted: 26 August 2013 / Published online: 8 September 2013

(C) The Author(s) 2013. This article is published with open access at Springerlink.com

\begin{abstract}
Background Vitreoretinal surgeons have been slow to adopt the use of spectral filters for endoillumination to reduce retinal light toxicity. This study shows that spectral filters can be used without a loss in color contrast during brilliant blue G chromovitrectomy.
\end{abstract}

\begin{abstract}
No funding from governmental or non-governmental institutions was received for this study. Funding by Bausch \& Lomb ${ }^{\mathrm{TM}}$ Surgical, Aliso Viejo, CA/USA, covered honoraria of an image analysis specialist who analyzed video material after it had been labeled by the corresponding author. The authors had full control of all primary data and they agree to allow Graefes Archive for Clinical and Experimental Ophthalmology to review their data upon request. None of the authors holds any financial or proprietary interest in the material presented in this article. At all times, the tenets of the Declaration of Helsinki were observed. Prospective approval of the study was obtained from the local Institutional Human Experimentation Committee (Ethikkommission beider Basel Nr. 279/11). Due to the observational character of the study, informed patient consent was not required. The study was registered at Clinicaltrials.gov under the (NCT01485575).
\end{abstract}

\author{
P. B. Henrich \\ Department of Ophthalmology, University of Basel, \\ Basel, Switzerland \\ C. Valmaggia $\cdot$ C. Lang \\ Department of Ophthalmology, Cantonal hospital, \\ St. Gallen, Switzerland \\ P. C. Cattin \\ Medical Image Analysis Center, University of Basel, \\ Basel, Switzerland \\ P. B. Henrich $(\bowtie)$ \\ Department of Ophthalmology, University Hospital Basel, \\ Switzerland, P.O. Box, CH-4012, Basel, Switzerland \\ e-mail: Paul.Henrich@usb.ch
}

Methods To evaluate the influence of intra operative spectral light filters on perceivable contrast during Brilliant Blue $\mathrm{G}$ chromovitrectomy, a prospective, observational clinical study was carried out on 59 consecutive Brilliant Blue G chromovitrectomy interventions in 59 patients admitted for macular holes, macular pucker or vitreomacular traction syndromes. Subsequent to peeling of the internal limiting membrane, six different illumination modes were enabled consecutively: mercury vapor, mercury vapor/xenon, and xenon followed by xenon combined with an amber, green or yellow spectral filter. Main outcome measure was the chromaticity spread between stained internal limiting membrane and unstained retina as a measure for the color contrast perceived by the human eye. Results Mean chromaticity scores were similar for all light sources: mercury vapor 7.97 , mercury vapor/xenon 7.96 $(p=0.96)$, and xenon $7.41(p=0.55)$. Compared to xenon, the additional use of endoillumination spectral filters did not change contrast recognizability: Chromaticity scores were 9.38 for the amber filter $(p=0.13), 6.63$ for the green and 7.02 for the yellow filter ( $p=0.37$ and 0.64 , respectively). When comparing the different filters head-to-head, the amber filter was superior to the green filter $(p=0.03)$, while the yellow was intermediate and not significantly different from either the amber $(p=0.08)$ or the green filter $(p=0.51)$.

Conclusions Color contrast perceptibility during Brilliant Blue $\mathrm{G}$ assisted chromovitrectomy is similar with mercury vapor, mercury vapor/xenon or xenon light sources. Spectral filters do not decrease color contrast recognizability. Head-tohead comparison shows a significant advantage for the amber over the green filter with respect to contrast generation, the yellow filter is intermediate. As spectral filters are known to greatly reduce retinal light toxicity, we suggest donor eye studies to validate whether the amber filter should be generally recommended for Brilliant Blue $\mathrm{G}$ chromovitrectomy. 
Key words Chromovitrectomy $\cdot$ Chromodissection $\cdot$ Brilliant Blue $\mathrm{G} \cdot$ Endoillumination $\cdot$ Spectral filters $\cdot$ Contrast generation

\section{Introduction}

Light-induced toxicity to the retina and the retinal pigment epithelium (RPE) from fiberoptic endoillumination during vitrectomy has been well established [1-3]. This phenomenon is of particular clinical relevance in macular surgery [4]. One of the most frequent interventions in macular surgery is the removal of the internal limiting membrane (ILM). The use of vital dyes in order to stain the ILM and improve its visibility is frequently referred to as chromovitrectomy [5] or chromodissection[6] and is now accepted as a standard treatment for diseases related to vitreomacular traction [7]. An additional increase in cellular susceptibility to light toxicity may develop through the use of several vital dyes $[8,9]$, including Brilliant Blue G (BBG), although clinical significance has only been demonstrated for indocyanine G (ICG) [8]. Brilliant Blue G is currently the only ILM-specific vital dye which is approved for intravitreal use in the European Union. Spectral filters have been proposed for several decades to significantly reduce retinal and retinal pigment epithelium light toxicity through the reduction of the endoillumination short-wavelength spectrum $[4,10]$. Spectral filters, also referred to as pass filters, are featured in many commercially available endoilluminator devices but only few surgeons rely on their use despite a growing body of evidence for their protective effect $[3,4,11-13]$. The influence of pass filters on the surgeon's intraoperative visual discrimination has been a matter of speculation for many years [4]. Objective clinical analyses are not available to our knowledge. We suspect that surgeons are concerned that the use of spectral filters might reduce visual control during chromovitrectomy. On the other hand, a preclinical examination recently suggested that spectral filters as well as polarizing filters may in fact improve intraoperative color contrast [14]. The present clinical study intends to objectify the influence of spectral light filters on intraoperative recognizability of the contrast between the stained ILM and unstained retina in BBG chromovitrectomy.

\section{Methods}

In a prospective, observational clinical study, we analyzed 59 consecutive chromovitrectomy interventions in 59 patients from one study center. Patients had been admitted for macular holes, macular pucker or vitreomacular traction syndromes. Exclusion criteria included patient age under 18 years, previous chromovitrectomies within the preceding 6 months and the use of additional vital dyes during the same intervention, including the application of trypan blue in order to visualize epiretinal material. Subjects were also excluded if videos did not permit the identification of posterior pole anatomic landmarks, including the optic disc, vascular arcades and the macula due to poor video quality or artifacts.

All patients underwent routine 23-gauge vitrectomy, performed by two surgeons (CV, CL) using the Stellaris PC surgical platform (Bausch \& Lomb ${ }^{\mathrm{TM}}$ Surgical, Aliso Viejo, CA/USA). This device is commercially equipped with two different integrated light sources for endoillumination: one xenon light source and one combined mercury and xenon light source. It also disposes of green, yellow and amber spectral filters which, if activated with a switch, filter the emission spectrum generated by the xenon light source before the light is conveyed to the endoillumination hand piece. The operating theater setup was also fitted with an additional external mercury vapor light source (Photon II $^{\mathrm{TM}}$, Synergetics ${ }^{\mathrm{TM}}$, Inc. O'Fallon, MO/USA). The relative spectral output for the examined light sources and filters are depicted in Fig. 1 (mercury vapor and xenon light) and in Fig. 2 (green, yellow and amber filter), respectively. Following complete posterior vitreous detachment, $0.5 \mathrm{ml}$ of a heavier than water BBG solution from ready-to-use vials (Brilliant Peel ${ }^{\circledR}$, Geuder AG, Heidelberg, Germany) were injected into the vitreous cavity at a concentration of $0.25 \mathrm{mg} / \mathrm{ml}$, with clearance occurring after $10 \mathrm{~s}$. The distance from the posterior retinal pole during injection was approximately $3 \mathrm{~mm}$. According to the manufacturer, this $0.25 \mathrm{~g} / 1$ solution of heavier than water BBG has an absorption maximum of $584.0 \mathrm{~nm}$ at a $\mathrm{pH}$ of 7.52 and an osmolarity of $306 \mathrm{mOsm} / \mathrm{kg} \mathrm{H} 2 \mathrm{O}$. Its specific weight is $1.017-1.019 \mathrm{~g} / \mathrm{cm}^{3}$. BBG is approved for intravitreal use in the European Union but not in the United States.

Mechanical removal of the ILM was performed under mercury vapor endoillumination (Photon II ${ }^{\mathrm{TM}}$, Synergetics ${ }^{\mathrm{TM}}$, Inc. O'Fallon, MO/USA) in combination with a widefield diffusion light pipe (56.21.23P, Synergetics ${ }^{\mathrm{TM}}$, Inc. O'Fallon, MO/USA). After completion of the peeling process, optimum lighting was sought for digital video recordings of the posterior pole. The same region was consecutively taped under 6 different illumination modes: Following mercury vapor illumination, both the mercury vapor/xenon and the xenon light sources incorporated in the surgical platform were enabled and recorded in combination with a wide field endoillumination cannula (BL5823 wide field illuminator, Bausch \& Lomb ${ }^{\mathrm{TM}}$ surgical, Aliso Viejo, CA/USA). Subsequently, the built-in amber, green and yellow filters were swung in successively and recorded in combination with xenon illumination.

For video recordings, a Panasonic LQ-MD800E digital recording system (Panasonic Corporation, Osaka, Japan) with a Leica 2 Type 10446585 video camera, attached to a Leica M841 Ophthalmic Microscope (Leica Microsystems GmbH, Wetzlar, Germany) was used. Routine exposure and calibration alignments of the recording system were performed at the beginning of each intervention, adjusting the white balance of the recording 

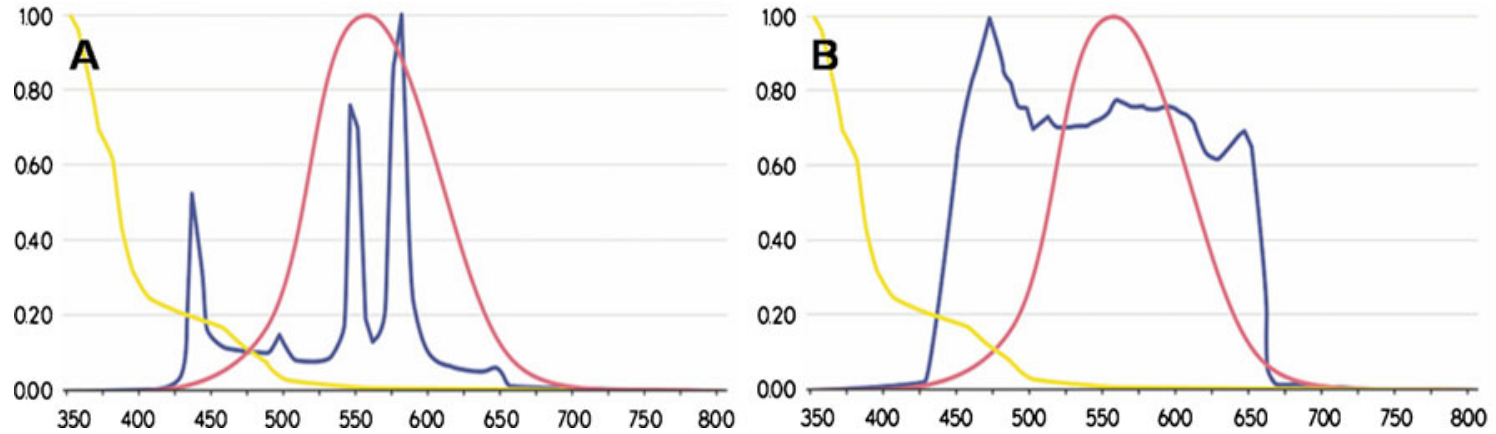

Fig. 1 Blue line: Mercury vapor (a) and xenon (b) endoillumination relative spectral output. Pink line: Relative photopic eye response curve. Yellow line: Relative aphakic hazard [3]. The ordinate displays a decimal division, the abscissa the wavelength in nanometers

system to a standardized balancing screen (XpoBalance ${ }^{\circledR}$, Lastolight Ltd., Coalville, Leics, United Kingdom).

Main outcome measure was the chromaticity spread between stained ILM and the unstained retina based on a method
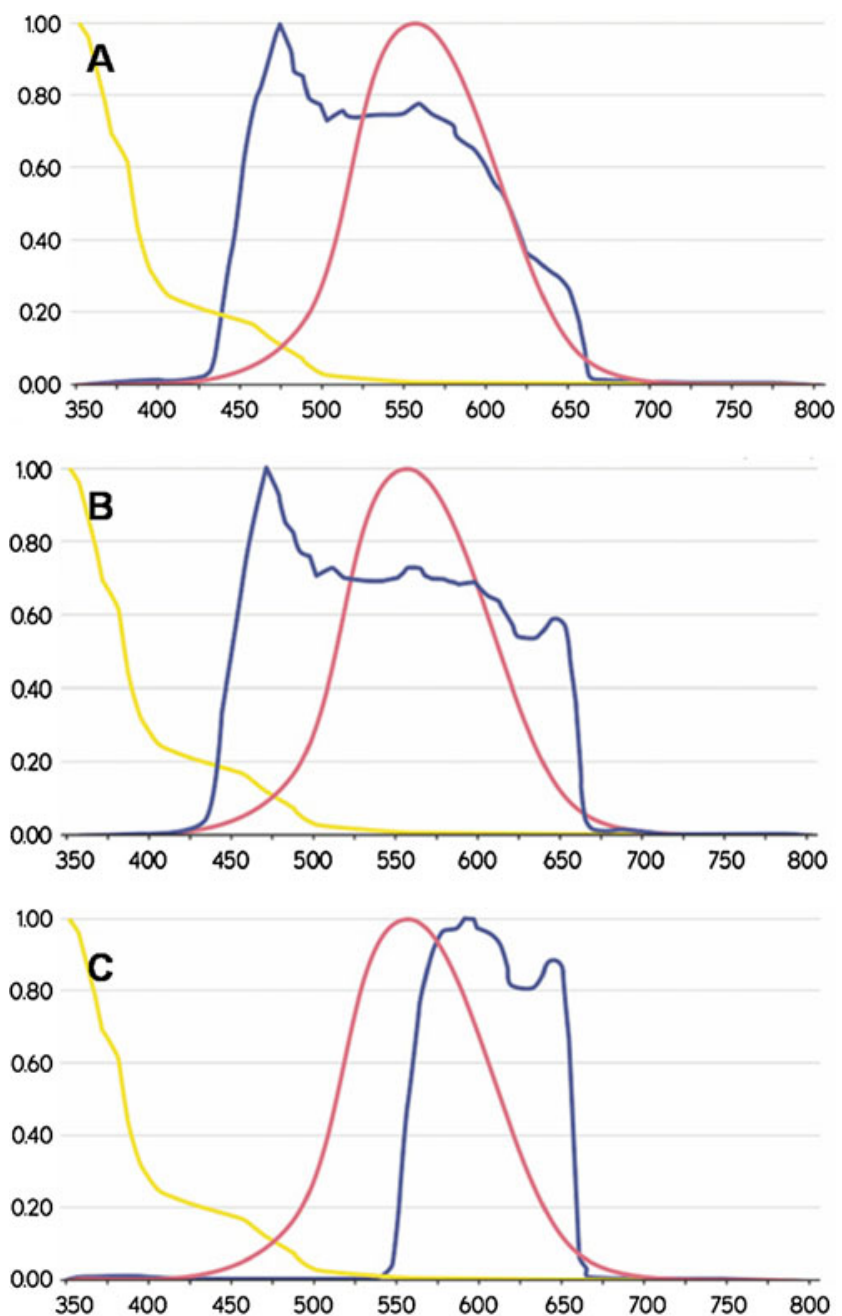

Fig. 2 Blue line: xenon+green filter (a) xenon+yellow filter (b) and xenon + amber filter $(\mathbf{c})$ endoillumination relative spectral output. Pink line: Relative photopic eye response curve. Yellow line: Relative aphakic hazard [3]. The ordinate displays a decimal division, the abscissa the wavelength in nanometers we now refer to as chromaticity analysis. The methodology is described in detail elsewhere [15]. Its objective is to quantify the color contrast between the stained ILM and unstained retina as it is perceived by the human eye. Briefly, individual still images without imaging artifacts were selected from each video. Two regions of interest (ROI) were marked in each of the images near the posterior pole and within the temporal vascular arcades by a vitreoretinal surgeon $(\mathrm{PBH})$ : One of the ROIs was plotted in an area with maximally stained ILM. A second ROI of similar extension was selected in an area where the ILM had already been removed during the course of the operation (Figs. 3 and 4). Based on the wavelengths captured by the charge-coupled device (CCD) of the camera, a custom made software tool programmed in MATLAB (Version R2007b) calculated the average color of each of the two ROIs. The averaged wavelengths were projected into the CIELAB (CIE 1976 L*,a*,b*) color space, a vector space diagram, in which all visible colors are plotted according to their discriminability by the human eye. It is based on empirical analyses of human visual sensitivities to color differences from the 1940s [16]. The original diagram featured multiple ellipsoid regions. Each ellipse contained a multitude of colors indistinguishable from the color in the center of the ellipse for the human eye. However, size and orientation of the regions varied depending on the center color, which was felt to be unpractical. An attempt to create a less distorted representation lead to an arrangement where the ellipses were converted into equidistant circles of colors indistinguishable from the color in the center of each circle. Distances between individual colors were now directly proportional to their discriminability by the human visual system. Distances within the CIELAB space can be regarded as a direct measure for the strength of perceivable color contrast [17]. They can be expressed quantitatively as chromaticity scores. Within the CIELAB color space, each color is uniquely defined by the $\mathrm{L}^{*}, \mathrm{a}^{*}$ and $\mathrm{b}^{*}$ values. To ensure invariance to different lighting, the lightness $\mathrm{L}^{*}$ was excluded from our calculations. Chromaticity scores are, thus, given by the Euclidean distance of the two colors in the $\mathrm{a}^{*} / \mathrm{b}^{*}$ plane and are in arbitrary units. Altogether, 324 chromaticity measurements were performed. 
Fig. 3 Screenshot displaying the same region of the fundus with plotted regions of interest (ROIs; long arrow) under different lighting: mercury-vapor (a), mercury-vapor-xenon (b) and xenon (c). The border between the stained ILM and the unstained underlying retina can be clearly appreciated (short arrow)
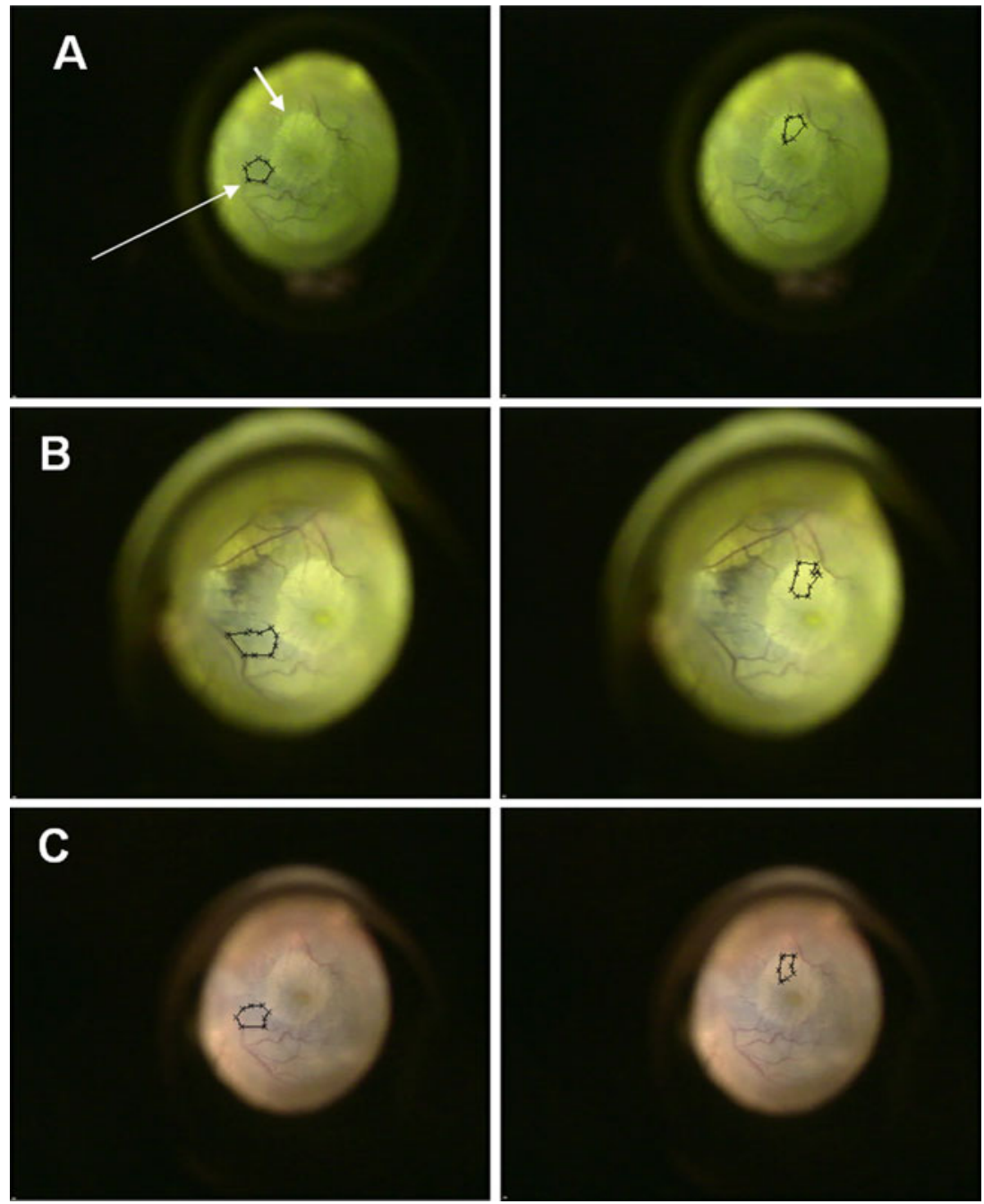

Statistically significant levels were calculated using the software package R Version 2.12.1 [18]. Normality of the distributions was tested with the Kolmogorof-Smirnov test and all our measurements were normally distributed.

To compare subgroup mean values one way ANOVA's were performed. Results were presented as F-values of the ANOVAs with corresponding $\mathrm{p}$-values. In case of nonsignificance, no further comparisons of subgroups were needed. In case of significance, subsequent pairwise t-tests would have been performed. To adjust $\mathrm{p}$-values for multiple comparison, the Holmes correction method would have been applied. A p-value smaller than 0.05 was considered statistically significant for all $t$-tests and ANOVAs in this study.

\section{Results}

A total of 59 consecutive chromovitrectomy interventions in 59 patients from one study center were analyzed. Indications for chromovitrectomy included macular holes $(N=18)$, macular pucker $(N=38)$ or vitreomacular traction syndromes $(N=3) ; 36$ right eyes and 23 left eyes were included. Patient age ranged from 45 to 82 years (median 72), and 27 patients were female while 32 were male. All patients were pseudophakic. Mean chromaticity scores were similar for all light sources: Mercury vapor 7.97, mercury vapor/xenon 7.96 ( $p=0.96$ compared to mercury vapor), and xenon 7.41 ( $p=0.55$ compared to mercury vapor). Compared to xenon, the additional use of endoillumination spectral filters did not change contrast recognizability. Chromaticity scores were 9.38 for the amber filter ( $p=0.13), 6.63$ for the green and 7.02 for the yellow filter ( $p=0.37$ and 0.64 , respectively). When comparing color contrast recognizability with the different filters head-to-head, the amber filter was superior to the green filter $(p=0.03)$. The yellow filter was intermediate compared to the amber $(p=0.08)$ or the green filter $(p=0.51)$ (Fig. 5). Chromaticity data for all subgroups revealed a Gaussian distribution. Comparison of disease subgroup mean values revealed a p-value of 0.21 . 
Fig. 4 Screenshot displaying the same region of the fundus with plotted regions of interest (ROIs; long arrow) under xenon lighting combined with Amber (a), green (b) and yellow spectral filters (c). The border between the stained ILM and the unstained underlying retina can be clearly appreciated (short arrow)
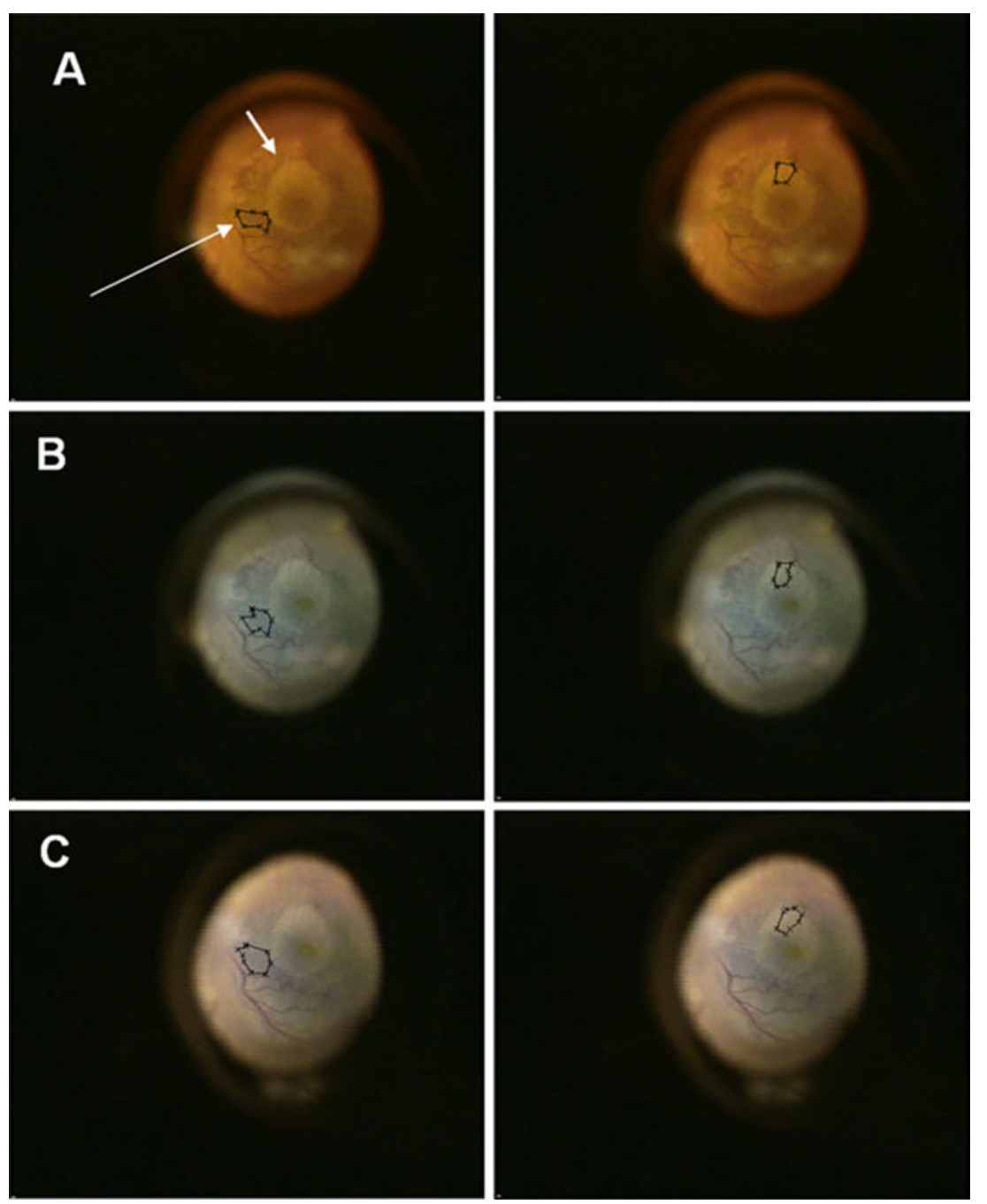

\section{Discussion}

Visual discrimination is essential for successful macular surgery. Stronger fiberoptic light sources and vital dyes have increased the ease, thoroughness, and safety of chromovitrectomy interventions [7]. Stronger light sources and the use of vital dyes may also increase cellular light toxicity, however, through increased light exposure and phototoxicity [9]. Light toxicity may be counteracted through the elimination of particularly hazardous wavelengths from the emission spectrum before entering the eye through the fiberoptic system. This approach involves the use of spectral filters and has been established for many years [4]. Many modern endoillumination devices feature a variety of built-in pass filters, yet vitreoretinal surgeons have been slow to adopt their use. When considering the established protective effects of spectral filters [3, 4, 11-13], a likely explanation for the surgeons' reservations lies in a concern over possible interference with intraoperative visual control. The relatively scarce literature on the matter does not, however, endorse such reservations; objective clinical analyses are not available, but surgeons have reported, in fact, improved visual discrimination with yellow filters $[4,19]$ and recently a preclinical study confirmed that the contrast between the stained ILM and unstained retina may be improved through the use of a pass filter which eliminates all wavelengths shorter than $500 \mathrm{~nm}$ during BBG chromovitrectomy on post-mortem porcine eyes [14]. The favorable effect of tinted glasses on visual function has been known since ancient times [20]. Yellow and amber filters have been described to increase contrast perception by a reduction of ocular media light scatter and decreased chromatic aberration [20]. Visual acuity studies have shown, that yellow and amber tinted lenses may increase contrast sensitivity to white on blue gratings 1.23 and 1.43 -fold, respectively: blue light is absorbed, reducing the luminance of the blue surface and hence improving the apparent contrast of the lighter objects [21].

The capacity of the human visual system to recognize color contrasts is not linear; some color contrasts can be differentiated more easily than others. In order to allow our results to meaningfully describe the surgeon's capacity to perceive the color contrast between the stained ILM and the unstained 
Fig. 5 Mercury vapor, xenonmercury and xenon light sources produce comparable contrasts in BBG chromovitrectomy. The use of intraoperative light filters also does not significantly alter BBG performance with respect to contrast formation. The ordinate displays the CIELAB score. The upper and lower margins of the boxes in this standard box-andwhisker diagram represent the 25 th and the 75 th, the central line inside the box the 50th percentile (median). The whiskers mark the minimum and the maximum, with some outliers plotted as small circles

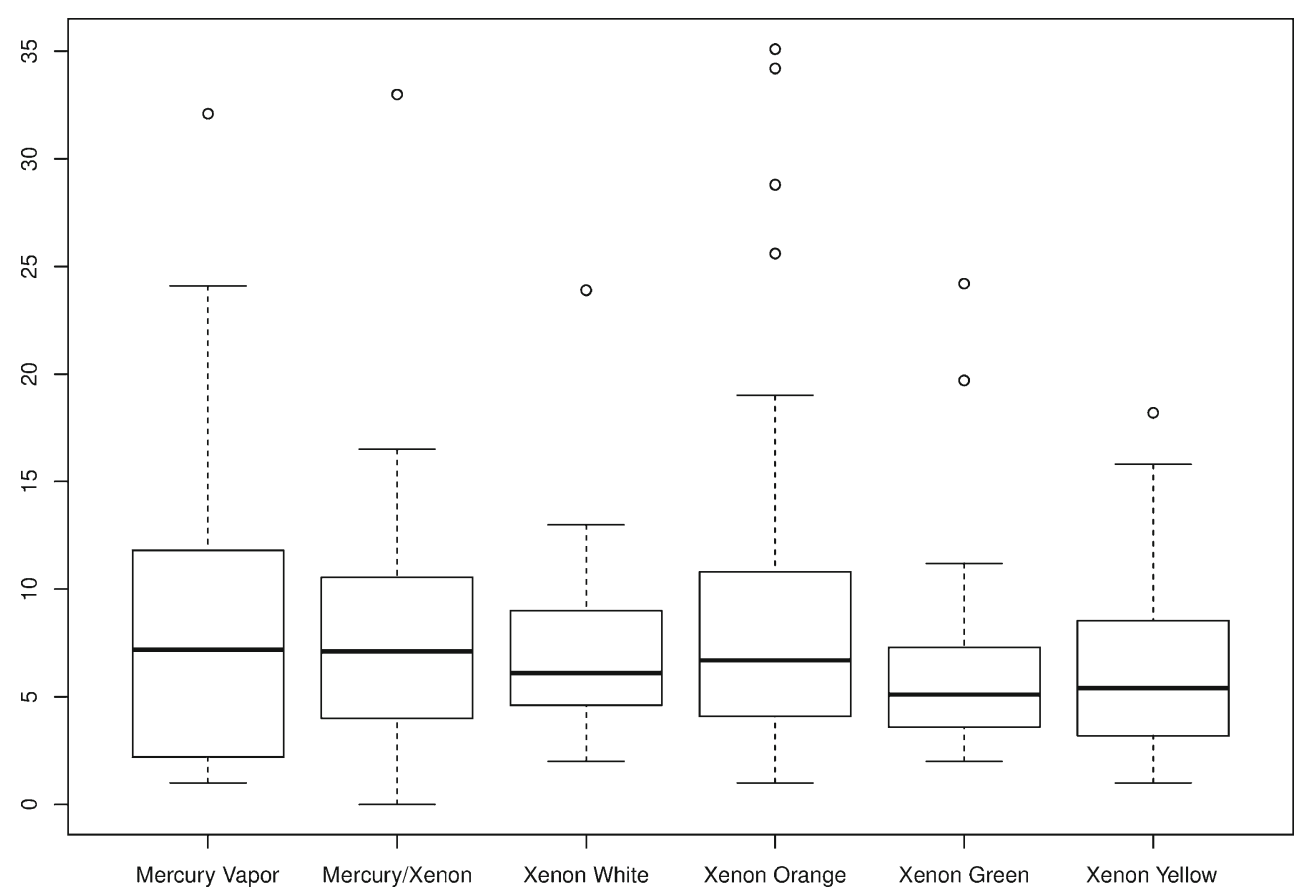

retina, we chose the chromaticity analysis method [22]. This method first calculates the average color contained in the two ROIs selected in each image. The averaged wavelengths are

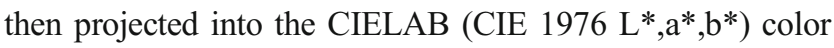
space. Distances within the CIELAB space can be regarded as a direct measure for the strength of perceivable color contrast [17]. They can be expressed quantitatively as chromaticity scores. The higher the chromaticity score the better the two colors can be differentiated by the observer.

Light-induced retinal toxicity is determined by the emission spectrum, power, and duration of application of endoillumination as well as by the distance between the light pipe and the retina [2]. Short wavelength irradiation, such as blue and ultraviolet rays, is particularly hazardous [3] as wavelengths under $400 \mathrm{~nm}$ have been shown to compromise photoreceptor function in addition to mediating the RPE damage seen across the entire spectrum of visible light exposure [2]. Overall phototoxic retinal exposure is 10 times higher at $435 \mathrm{~nm}$ compared to a $500 \mathrm{~nm}$ wavelength [23].

Different light sources feature distinct emission spectra with diverging short wavelength spectral radiances.

Photosensitizing dyes additionally prompt photochemical retinal damage by increasing levels of free radicals as an overlap of the endoilluminator emission spectrum and the vital dye absorbance spectrum provokes the absorbance of endoillumination light by the dye-stained retina. This phenomenon is particularly relevant in the case of ICG $[8,24]$. For several other vital dyes, including BBG, a decrease in cellular viability has been described in connection with the presence of visible light, although a clinically measurable effect has not been observed at conventional dosages [9].
Among the investigated endoillumination devices, xenon illumination, due to its emission spectrum, presents a higher phototoxicity hazard than mercury vapor light [3, 25] (Fig. 1). Exposure times to reach a safety limit defined by ISO 15004 2:2007 are 1.9 times shorter for xenon than for mercury vapor light (Table 1). The protective effects of spectral filters can also be expressed as an increment in exposure times necessary to reach the predefined ISO 15004-2:2007 safety limit (Table 1). Among the filters incorporated in the surgical platform used for the present clinical study, the amber filter allows the most resolute elimination of short wavelengths. The resulting reduction in light toxicity allows exposure times more than three times higher than white xenon light to reach the exposure limit. The protective effects are less pronounced for the yellow filter and even less so for the green filter.

Our results show that a diligent choice of the endoillumination light source and the activation of appropriate additional spectral filters allow operating with significantly reduced retinal and RPE light toxicity without compromising in terms of color contrast performance: While no difference with regards to contrast performance was observed in our study, mercury vapor light sources have a more favorable light toxicity profile than xenon light sources. None of the incorporated spectral filters significantly changed color contrast discriminability compared to white xenon light. When comparing the different filters head-to-head, the amber filter was significantly superior to the green filter. Contrast strength registered with the use of the yellow filter was in between that of both other filters, statistical significance was not reached.

Comparatively good contrast values for the amber filter are in line with earlier observations that filters which reduce the 
Table 1 Time to exceed $10 \mathrm{~J} / \mathrm{cm}^{2}$ weighted radiant exposure guideline, in minutes

\begin{tabular}{|c|c|c|c|c|c|c|c|c|}
\hline \multirow{2}{*}{$\begin{array}{l}\text { Lamp: } \\
\text { Spectral filter: }\end{array}$} & \multicolumn{4}{|c|}{ Mercury } & \multicolumn{4}{|l|}{ Xenon } \\
\hline & None & Amber & Green & Yellow & None & Amber & Green & Yellow \\
\hline \multicolumn{9}{|l|}{ Setting (\%) } \\
\hline 100 & 29 & $>120$ & 33 & 44 & 16 & $>120$ & 18 & 23 \\
\hline 60 & 49 & $>120$ & 56 & 73 & 26 & $>120$ & 30 & 39 \\
\hline 50 & 58 & $>120$ & 67 & 87 & 32 & $>120$ & 36 & 46 \\
\hline 40 (=default) & 73 & $>120$ & 84 & 109 & 39 & $>120$ & 45 & 58 \\
\hline 30 & 97 & $>120$ & 112 & $>120$ & 53 & $>120$ & 60 & 77 \\
\hline 20 & $>120$ & $>120$ & $>120$ & $>120$ & 79 & $>120$ & 89 & 116 \\
\hline 10 & $>120$ & $>120$ & $>120$ & $>120$ & $>120$ & $>120$ & $>120$ & $>120$ \\
\hline
\end{tabular}

Table [26]: Time to exceed the $10 \mathrm{~J} / \mathrm{cm} 2$ ISO 15004-2:2007 weighted radiant exposure limit in minutes with the two different light sources featured in the Bausch \& Lomb Stellaris PC surgical platform. The photo toxicity hazard is lower with mercury vapor than with xenon illumination and can be gradually further reduced with the use of the green, yellow and amber spectral filter, respectively. Note: The exposure from all light sources is cumulative and additive

short wavelength emission spectrum increase color contrast sensitivity by reducing ocular media light scatter and chromatic aberration and with a preclinical analyses which show that pass filters may allow excellent contrast performance [14]. Blue light absorption leading to improved discriminability of surrounding lighter structures may also play a role. The favorable performance of the amber filter is also endorsed by the finding that its spectrum coincides more than that of any other tested filter or light source with the BBG absorption spectrum. Complete overlap of the spectra would generate a binary image where only unstained tissue would reflect the amber light, thus, maximizing contrast, although at the expense of reduced luminance. Generally, the reduction in retinal photo toxicity needs to be weighed against lower overall levels of luminance and reduced color discriminability [11, $14,21]$. Luminance has been described to drop to $14-71 \%$ through the use of a pass filter with a cut-off at $500 \mathrm{~nm}$ in a preclinical study, although illumination was still considered sufficient for ILM removal [14]. Luminance was excluded in the present study, as it is subject to interference from inconsistent angles of illumination and variable distance between the illumination probe and the retinal surface. The cut-off of the amber filter of roughly $550 \mathrm{~nm}$ is similar to the one used in the preclinical study so that a similar reduction in luminance is to be expected. We speculate that reduced luminance contrast, apart from a subjective preference for white light may, in part, explain the sluggish adoption of spectral endoillumination light filters among vitreoretinal surgeons. Donor eye studies are suggested to validate the feasibility of ILM removal under amber filter endoillumination, before a general recommendation for its routine use can be made.

Spectral filter options are also included in a number of other commercially available endoillumination light sources. Similar effects on retinal light toxicity and color contrast can be expected, although variations in the light spectrum suggest that our results should not be directly extrapolated to other devices. Presently, apart from the Stellaris PC surgical platform (Bausch \& Lomb ${ }^{\mathrm{TM}}$ surgical, Aliso Viejo, CA/USA) the Brightstar Illumination System (D.O.R.C. Dutch Ophthalmic Research Center International B.V. Zuidland, The Netherlands; cut-offs at $435 \mathrm{~nm}, 475 \mathrm{~nm}, 515 \mathrm{~nm}$ ), and the Synergetics Photon 1 and 2 (Synergetics Inc., O'Fallon, Missouri, USA; cut-offs at $485 \mathrm{~nm}$ ) all offer filter options.

Limitations of the methodology include the fact that the type of stained tissue, e.g. ILM vs. epiretinal material, was not monitored histologically and that the extension of analyzed retinal surface was not standardized per patient. ROIs were chosen manually by a retinal surgeon in an area with maximum contrast in each image: The results reflect the color contrast of areas with highest contrast according to the perception of the interpreting retinal surgeon, not an average of the posterior pole. We believe that the measurement of highest contrast may be clinically more meaningful than the posterior pole average; however, as the difficult elevation of the first ILM flap is generally performed in an area with optimum staining. Also, the comparison of areas with maximum contrasts minimizes bias by epiretinal material.

The patient population used in this study consists of a group of three related diagnoses, whose configurations might influence staining characteristics. However, ANOVA subgroup variance analyses showed a p-value of 0.21 , revealing that staining was not affected by the underlying pathology.

\section{Conclusions}

Color contrasts between the ILM and the underlying retina perceived by the human eye during BBG-assisted chromovitrectomy are similar with the use of mercury vapor, mercury vapor/xenon and xenon light sources. The use of spectral filters 
also does not significantly alter color contrast recognizability compared to white xenon light. When comparing different filters head-to-head, the amber filter produces significantly better color contrasts than the green filter, while yellow filter performance is intermediate with no statistical significance. The amber filter coincidentally offers higher protection from light toxicity than the yellow or the green filter.

In the context of this prospective clinical analysis, it is suggested that for the choice of the light source and spectral filter in BBG-assisted chromovitrectomy, light toxicity should be taken into account, favoring mercury vapor illumination and the use of the amber filter. Donor eye studies are needed to validate practicability, before a general recommendation can be made.

Contributions of the authors: Design and conduct of the study (PBH, PC); data collection ( $\mathrm{PBH}, \mathrm{CV}, \mathrm{CL}$ ), management $(\mathrm{PBH})$, analysis and interpretation of the data $(\mathrm{PBH}$, $\mathrm{PC})$; preparation $(\mathrm{PBH})$, review ( $\mathrm{PBH}, \mathrm{CV}, \mathrm{CL}, \mathrm{PC}$ ), or approval (PC) of the manuscript. None of the authors discloses any conflict of interest with the material presented in this article. Word count: 2978.

Open Access This article is distributed under the terms of the Creative Commons Attribution License which permits any use, distribution, and reproduction in any medium, provided the original author(s) and the source are credited.

\section{References}

1. Fuller D, Machemer R, Knighton RW (1978) Retinal damage produced by intraocular fiber optic light. Am J Ophthalmol 85:519-537

2. Costa Ede P, Rodrigues EB, Farah ME, Dib E, Penha F, Magalhaes O Jr, Furlani BA, Lima Filho AA, de Miranda A, Maia M (2009) Vital dyes and light sources for chromovitrectomy: Comparative assessment of osmolarity, $\mathrm{pH}$, and spectrophotometry. Invest Ophthalmol Vis Sci 50:385-391

3. van den Biesen PR, Berenschot T, Verdaasdonk RM, van Weelden H, van Norren D (2000) Endoillumination during vitrectomy and phototoxicity thresholds. Br J Ophthalmol 84:1372-1375

4. Meyers SM, Bonner RF (1982) Yellow filter to decrease the risk of light damage to the retina during vitrectomy. Am J Ophthalmol 94:677

5. Rodrigues EB, Meyer CH, Kroll P (2005) Chromovitrectomy: A new field in vitreoretinal surgery. Graefes Arch Clin Exp Ophthalmol 243: 291-293

6. Kicova N, Bertelmann T, Irle S, Sekundo W, Mennel S (2012) Evaluation of a posterior vitreous detachment: A comparison of biomicroscopy, B-scan ultrasonography and optical coherence tomography to surgical findings with chromodissection. Acta Ophthalmol 90:e264-268

7. Henrich PB, Haritoglou C, Meyer P, Ferreira PR, Schotzau A, Katamay R, Josifova T, Schneider U, Flammer J, Priglinger S
(2009) Anatomical and functional outcome in brilliant blue G assisted chromovitrectomy. Acta Ophthalmol 23:23

8. Gandorfer A, Haritoglou C, Kampik A (2008) Toxicity of indocyanine green in vitreoretinal surgery. Dev Ophthalmol 42:69-81

9. Morales MC, Freire V, Asumendi A, Araiz J, Herrera I, Castiella G, Corcostegui I, Corcostegui G (2010) Comparative effects of six intraocular vital dyes on retinal pigment epithelial cells. Invest Ophthalmol Vis Sci 2010:16

10. Sliney DH, Wolborsht ML (1980) Safety standards and measurement techniques for high intensity light sources. Vision Res 20:1133-1141

11. Kraushar MF, Harris MJ, Morse PH (1989) Monochromatic endoillumination for epimacular membrane surgery. Ophthalmic Surg 20:508-510

12. Yanagi Y (2008) Retinal phototoxicity from endoilluminators for vitrectomy. Nihon Ganka Gakkai Zasshi 112:975-983

13. Hillenkamp J, Dydykina S, Klettner A, Treumer F, Vasold R, Baumler W, Roider J (2010) Safety testing of indocyanine green with different surgical light sources and the protective effect of optical filters. Retina 30:1685-1691

14. Enaida H, Hachisuka Y, Yoshinaga Y, Ikeda Y, Hisatomi T, Yoshida S, Oshima Y, Kadonosono K, Ishibashi T (2013) Development and preclinical evaluation of a new viewing filter system to control reflection and enhance dye staining during vitrectomy. Graefes Arch Clin Exp Ophthalmol 251:441-451

15. Henrich PBPS, Haritoglou C, Rg S, Strauss RW, Schneider U, Josifova T, Cattin PC (2013) Quantification of contrast recognizability in sequential epiretinal membrane removal and internal limiting membrane peeling in trypan blue-assisted macular surgery. Retina 33(4):818-24

16. Macadam DL (1942) Visual sensitivities to color differences in daylight. Journal of the Optical Society of America 32:247-273

17. Kuehni RG (1976) Color-tolerance data and the tentative CIE 1976 L a b formula. J Opt Soc Am 66:497-500

18. Team RDC (2009) A language and environment for statistical computing. http://cran.r-project.org/bin/windows/base/old/2.12.1/. Accessed 05 April 2011

19. Meyers SM, Bonner RF (1982) Retinal irradiance from vitrectomy endoilluminators. Am J Ophthalmol 94:26-29

20. Rosenblum YZ, Zak PP, Ostrovsky MA, Smolyaninova IL, Bora EV, Dyadina UV, Trofimova NN, Aliyev AG (2000) Spectral filters in low-vision correction. Ophthalmic Physiol Opt 20:335-341

21. Wolffsohn JS, Cochrane AL, Khoo H, Yoshimitsu Y, Wu S (2000) Contrast is enhanced by yellow lenses because of selective reduction of short-wavelength light. Optom Vis Sci 77:73-81

22. Henrich PB, Priglinger SG, Haritoglou C, Josifova T, Ferreira PR, Strauss RW, Flammer J, Cattin PC (2011) Quantification of contrast recognizability during Brilliant Blue G-and indocyanine greenassisted chromovitrectomy. Invest Ophthalmol Vis Sci 52:43454349

23. Mittnacht D (2008) Emission limits and standards regarding endoilluminators. Ophthalmologe 105:911-915

24. Haritoglou C, Priglinger S, Gandorfer A, Welge-Lussen U, Kampik A (2005) Histology of the vitreoretinal interface after indocyanine green staining of the ILM, with illumination using a halogen and xenon light source. Invest Ophthalmol Vis Sci 46:1468-1472

25. Yanagi Y, Inoue Y, Jang WD, Kadonosono K (2006) A2e mediated phototoxic effects of endoilluminators. Br J Ophthalmol 90:229-232

26. Bausch \& Lomb surgical Incorporated R, NY (2011) Operator's Manual Addendum: 1/1 